\title{
Ossification in the region of the posterior longitudinal ligament as a cause of cervical myelopathy
}

\author{
L. BAKAY, H. L. CARES, AND R. J. SMITH \\ From the Division of Neurosurgery and Department of Radiology, State University of New York \\ at Buffalo, U.S.A.
}

In 1967, we operated on a patient for cervical cord compression caused by an unusual type of ectopic bone formation in the spinal canal which we tentatively identified as ossification within the posterior longitudinal ligament. Until that time we had believed that calcification of the vertebral ligaments is a phenomenon secondary to spondylosis (Oppenheimer, 1942) and does not cause symptoms by itself. However, about the same time, a description of no less than 18 similar cases was published by Onji, Akiyama, Shimomura, Ono, Hukuda, and Mizuno (1967). Additional cases were also found in Japan (Yokoi, 1963). It seems, therefore, that these cases, while probably rare, constitute a distinct pathological entity. Although calcification and ectopic bone formation have been described before in various ligaments of the spine (Oppenheimer, 1942; Cloward, 1967), they were found accidentally without giving rise to clinical symptoms. A search for similar cases was undertaken and six additional patients with such abnormalities were found.

\section{CASE REPORTS}

\section{ECTOPIC BONE FORMATION}

CASE 1 M.N., a 60-year-old white woman, had a one year history of difficulty in walking. She also had chronic urinary incontinence which she attributed to a previous hysterectomy. Six weeks before admission, her gait became much worse and she began having severe neck pain, paraesthesia and weakness in both arms, particularly in the right arm. One week before admission, she became unable to walk.

Examination on admission showed an obese white female who was unable to stand or sit. Motion of the neck was limited, particularly in rotation and flexion. Both triceps muscles were weak, particularly the right one, but biceps function was good. Both flexion and extension at the wrist were weak; the right grip was especially poor. There was no voluntary motion in either lower extremity. Sensation to pin prick and light touch was decreased below the T-4 dermatome. Position sense was intact. Deep reflexes were brisk throughout, pathologically active on the right side. The radial periosteal reflex was inverted bilaterally and she had positive Babinski reflexes on both sides.

Radiographs revealed a mild spondylosis of the cervical spine with narrowing of the intervertebral spaces at $\mathrm{C}_{5-6}$ and $\mathrm{C}_{6-7}$ and minimal uncinate hypertrophy in the lower cervical spine. The intervertebral foramina were normal. A strip of bony density was located in the spinal canal anteriorly (Fig. 1). The superior end of this structure was pointed and the lower end was not clearly defined. The sagittal diameter of the osseous density at $\mathrm{C}_{3}$ vertebra was $5 \mathrm{~mm}$, the transverse diameter $6 \mathrm{~mm}$.

A better resolution of the bony density was obtained by laminography. It extended from the level of $C_{1}$ to $C_{5}$ vertebra and occupied the anterior half of the spinal

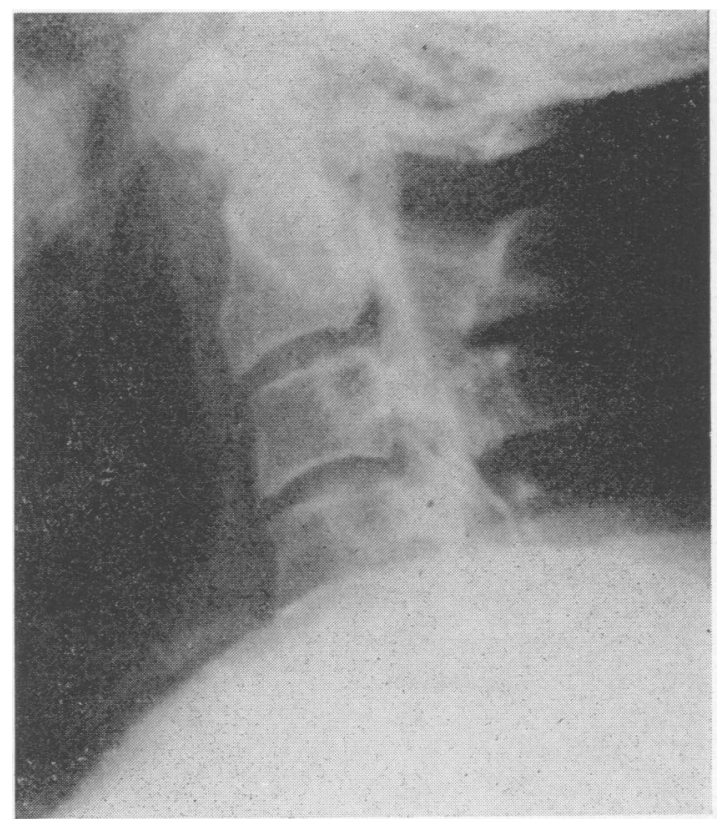

FIG. 1. Case 1. Lateral view of the cervical spine showing bone formation in the anterior spinal canal. 
canal. It was continuous with the posterior cortical surfaces of the bodies of $C_{2}$ and $C_{3}$ and possibly $C_{4}$ and $\mathrm{C}_{5}$ vertebrae.

Myelography showed that the spinal cord was compressed in its anteroposterior diameter and widened in its transverse diameter at the level of $\mathrm{C}_{2}$ to $\mathrm{C}_{4}$ vertebrae because of extradural encroachment on the neural canal by the bony bar (Fig. 2). The column of Pantopaque was markedly delayed in its passage past this area and the high cervical subarachnoid space filled only with difficulty.

A decompressive laminectomy was performed from $C_{1}$ to $C_{6}$. After the laminae of $C_{3}, C_{4}$, and $C_{5}$ vertebrae were removed, the dura appeared to be under tension and without pulsation. Removal of the $C_{2}$ lamina was followed by dural pulsation. After carrying the decompression on both sides as far laterally as possible, the dura was gently retracted medially. The epidural veins were engorged, but the nerve root sheaths appeared normal, although somewhat stretched due to the posterior displacement of the cord. The abnormal structure filled out the entire anterior half of the spinal canal. It had the appearance of solid, marble-hard bone. Its surface was smooth, but, apart from its lateral buttresses, it was tightly adherent to the dura. Decompression resulted in slackening and some posterior protrusion of the dura; it was not opened for fear of injury to the strongly compromised and kinked cord. The bony mass was not biopsied for the same reason.

Post-operatively, the patient became transiently worse

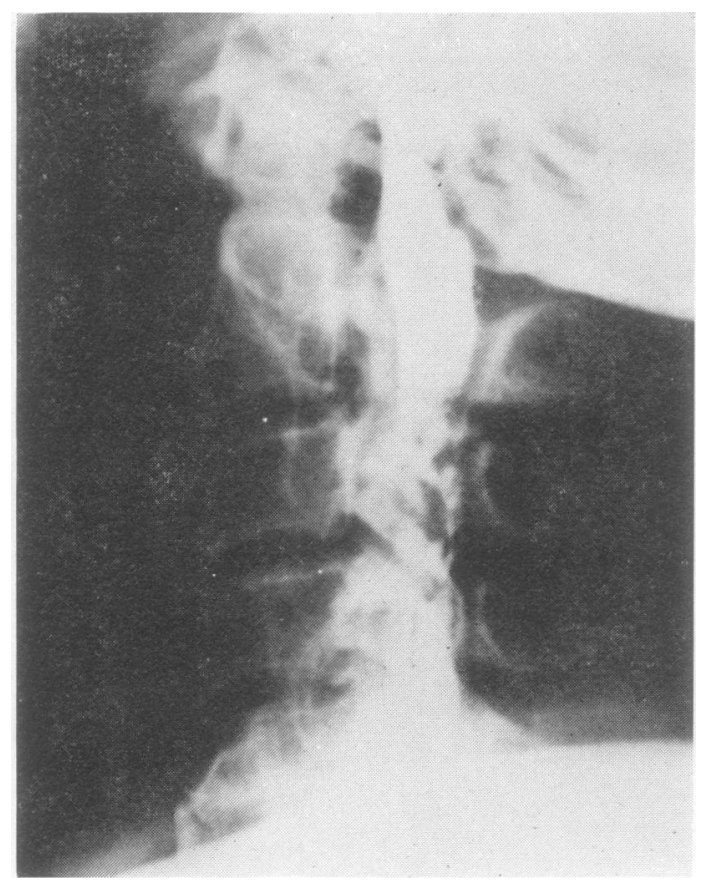

FIG. 2. Case 1. Lateral view of cervical myelogram. neurologically; her quadriparesis became more pronounced with complete paralysis of her right arm and leg. Two weeks later, the left side improved to the pre-operative level. However, two months later improvement was also noted in the right side. Five months after the operation there was considerable improvement in all four limbs. Although the right grip remained weak, the strength in the left arm returned to normal and she was able to feed herself. Muscle strength in the left leg returned to about $50 \%$ of normal and she was also able to lift her right leg against gravity.

CASE 2 A.A., a 55-year-old white woman, was admitted $\vec{F}$ with a three-month history of pain and inability to $\stackrel{9}{?}$ move her right shoulder. Six years earlier, she had a 0 thyroidectomy followed by a period of hypocalcaemia and 'seizures'. She was diagnosed as hypoparathyroid and treated with vitamin $D$.

There was limitation of neck motion in every direction. There was also restriction in mobility in the right shoulder, कै particularly in abduction, anterior flexion, and internal $\vec{\circ}$ rotation. Neurological examination was essentially negative; there was no weakness, sensory deficit, or reflex change in the right upper extremity and there was no evidence of long tract involvement of the spinal cord.

Radiographs revealed considerable spondylosis of $\omega_{\omega}$ various segments of the spinal column. The cervicap i spine revealed marked anterior spondylotic spurring but posterior spur formation was minimal and thes, was no encroachment upon the intervertebral foramina The most striking finding was the presence of a bong density in the anterior spinal canal between $\mathrm{C}_{2}$ and $C_{4}$ vertebrae. Its characteristics in the lateral view web very similar to that of case 1 (Fig. 3). On the front view, the mass appeared quite wide, reaching from $\overrightarrow{0} \cdot \overrightarrow{0}$ almost side to side across the width of the spinal canal.

Radiographs of the shoulder were negative. Although the pathological changes in the cervical spine suggested the possibility that the right shoulder pain was caused by radiculopathy rather than by a local articular disorder, the lack of characteristic neurological findings made this diagnosis uncertain. The patient refused myelography.

She was treated by cervical traction, infra-red heat to the shoulder and gradual mobilization. This resulted in improvement of her pain and slight increase in the range of motion in the right shoulder. A follow-up examination five years later revealed no significant change in the shoulder; the neurological status remained normal. The patient refused further radiographs.

\section{CALCIFICATION IN THE POSTERIOR LONGITUDINAL} LIGAMENT

CASE 3 W.B., a 72-year-old Negro male, died at the hospital from disseminated carcinoma of the lung. Radiographs showed marked spondylotic changes in the cervical spine from $C_{2}$ through $C_{7}$ vertebrae with $\frac{D}{O}$ narrowing of all the corresponding intervertebral discs and slight narrowing of all intervertebral foramina $N$ bilaterally. There was a thin sliver of calcification in the posterior longitudinal ligament, extending from $\mathrm{C}_{2} \mathrm{~N}$ to $C_{5}$ vertebrae. Neurological examination was negative. 


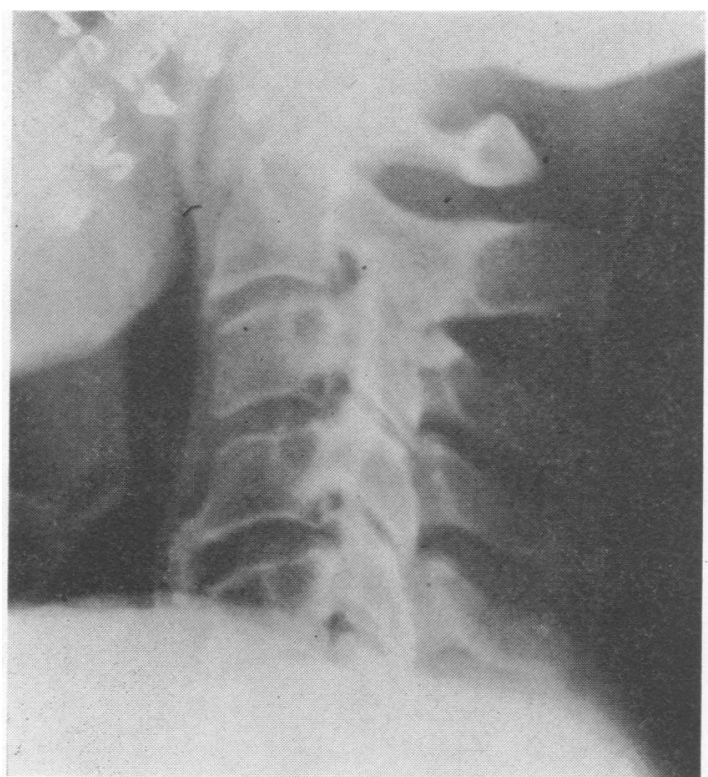

FIG. 3. Case 2. Abnormal bone formation in the anterior spinal canal between $C_{2}$ and $C_{4}$ vertebrae. Lateral view.

CASE 4 C.T., a 65-year-old white male, was diagnosed during his hospitalization as having amyotrophic lateral sclerosis. He was paraparetic with spastic lower extremities, bilateral Babinski reflexes, and bilateral facial palsy with pseudobulbar symptoms. Cervical spine films showed what appeased to be a calcified posterior longitudinal ligament extending from $C_{2}$ to $C_{4}$ vertebrae (Fig. 4).

CASE 5 H.W., a 51-year-old white male, was admitted with the main complaint of postural dizziness which particularly occurred when looking upward. It would subside upon flexing the neck. Neurological examination showed an essentially normal status, apart from the dizziness that was brought on by extending the neck. Radiographs of the spine showed severe changes compatible with rheumatoid arthritis. Although the bamboo appearance involved almost the entire spine, the changes were most pronounced in the cervical region. Here the bamboo-like appearance of the cervical spine with ossification of the spinal ligaments was quite striking. Lateral masses appeared to be partially fused, although apophyseal joints could be seen. Fusion occurred between the bodies of $\mathrm{C}_{4}, \mathrm{C}_{5}$, and $\mathrm{C}_{6}$ vertebrae, and very marked anterior osteophyte formation was seen between $C_{6}$ and $\mathrm{C}_{7}$ vertebrae. The cervical lordosis was fairly well preserved, but flexion and extension could not be carried out to any significant degree, the only motion having occurred at the $C_{1}$ and $C_{2}$ levels and between the occiput and $C_{1}$ vertebra. The abnormal posterior longitudinal ligamentous calcification appeared between $\mathrm{C}_{2}$ and $\mathrm{C}_{5}$ vertebrae (Fig. 5). The calcification was thicker in the higher segments and tapered toward its lower end.

CASE 6 J.W., an 85-year-old white male, had no history of neurological symptoms until the day of admission when he was found in his home lying on the floor unconscious. He was quadriplegic with a sensory level at $\mathrm{C}_{5}$ dermatome. The neck was painful. He had flaccid paralysis of all four limbs and an absence of tendon reflexes over the upper as well as lower extremities and abdominal reflexes. Babinski reflex was present on the left side; there was no plantar reflex on the right.

Radiographs of the cervical spine showed marked spondylosis and a rotated fracture-dislocation between $\mathrm{C}_{5}$ and $\mathrm{C}_{6}$ vertebrae. There was also a considerable calcification of the posterior longitudinal ligament which extended from $C_{2}$ to $C_{5}$ or $C_{6}$ vertebrae. On the lateral view, the diameter of this calcified structure was quite impressive; it encroached upon the spinal canal from the anterior direction, narrowing it to a considerable extent (Fig. 6). Upon considering the completeness of the motor and sensory deficit as well as the patient's age, conservative measures of treatment were applied.

CASE 7 M.L., a 65-year-old white male with no localizing neurological findings, but with advanced ankylosing spondylitis, showed atlanto-axial fusion with $C_{1}$ to $C_{2}$ calcification in the posterior longitudinal ligament.

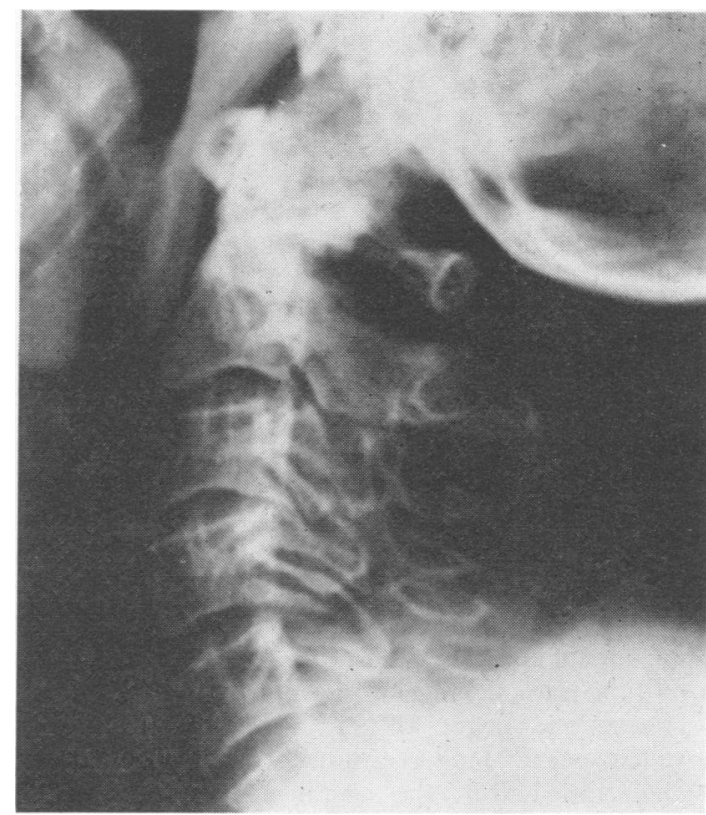

FIG. 4. Case 4. Lateral radiograph of the cervical spine reveals thin calcification of the posterior longitudinal ligament between $C_{2}$ and $C_{4}$ vertebrae. 


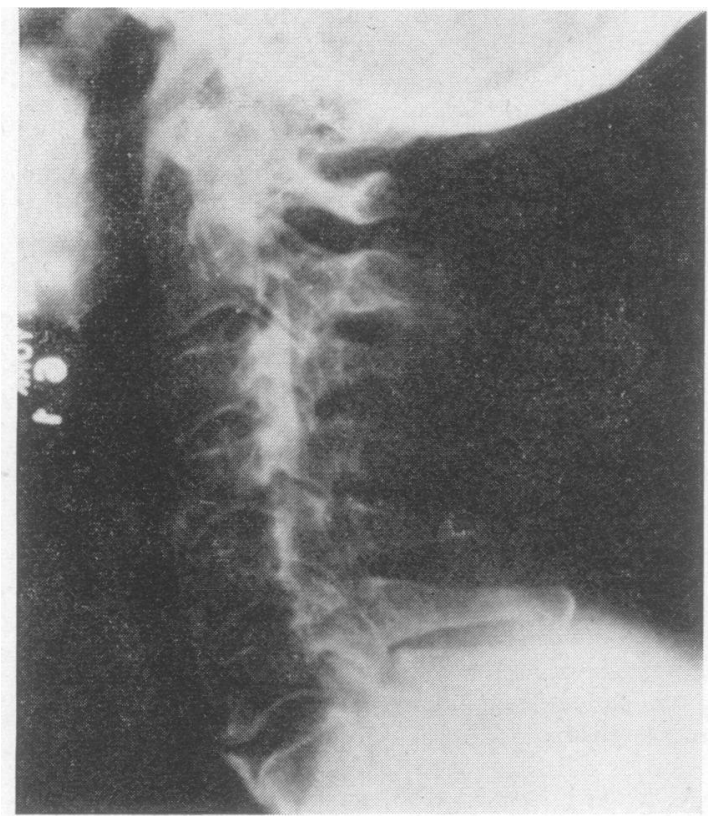

FIG. 5. Case 5. Lateral radiograph of the cervical spine shows extensive calcification of the posterior longitudinal ligament between $C_{2}$ and $C_{5}$ vertebrae as well as severe rheumatoid arthritis of the spine and osteoporosis of the vertebral bodies.

\section{DISCUSSION}

From a morphological point of view, there is a great range of variation in these cases from a thin layer of calcium deposition in the posterior longitudinal ligament to a thick, completely ossified, bony structure in the same anatomical region. The degree of associated spondylotic vertebral changes varies, but there seems to be a distinct entity of cases with ectopic bone formation without any or only minimal vertebral pathology. Our cases tended to be of two types. In the first group, exemplified by two cases (cases 1,2 ), a hefty slab of bone is present in the anterior spinal canal and there are either no spondylotic changes present in the cervical spine or only to such a degree as is not unusual among the randomly selected population of the patient's age.

The second group of patients consisted of five cases (cases 3 to 7 ) representing ordinary degenerative calcification within the confines of the posterior longitudinal ligament concomitant with other changes of marked cervical spondylosis. Although it is impossible to distinguish calcification from ossification radiographically a definite difference

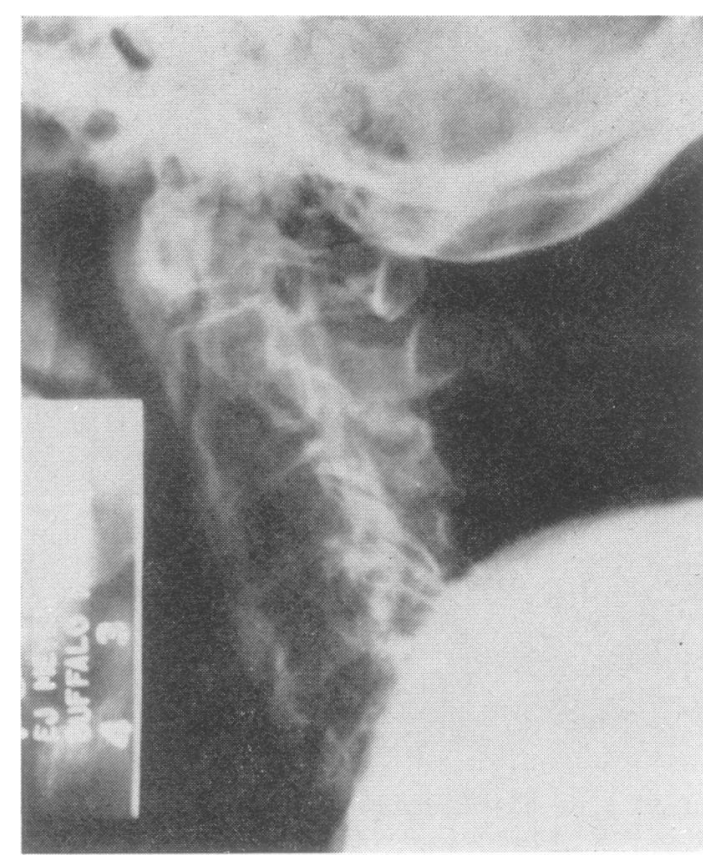

FIG. 6. Case 6. Lateral radiograph of the cervicat spine. Marked calcification of the posterior longitudina $\mathbb{Q}$ ligament in the presence of marked spondylosis.

in the appearance of this group from that of th first was noticed.

Symptomatic ossification in the spinal canal was first reported in large numbers in patients of Oriental, and particularly of Japanese, extraction (Yokoi, 1963;Onji et al., 1967). However, a later report is based on patients of Caucasian extraction (Minagi and Gronner, 1969). It still remains to be seen whether this condition is more prevalent in Japan than elsewhere. Onji et al. (1967) reported a frequency of $1.7 \%$ among their patients with cervical symptoms and a similar Japanese report by Yokoi (1963) calculated the frequency as being $1 \%$. At the same time, the survey of a large number of Cloward's patients (Onji et al., 1967) of Japanese ancestry failed to demonstrate such lesions.

Our cases, as well as those reported in the literature, represent patients in later life, with no striking sexual predilection. Grossly, the bar is composed of hard bone as described in case 1 , located between the posterior longitudinal ligament and the dura to which it is tightly adherent. These abnormalities have been identified histologically as varying from unorganized calcification to fully formed bone with fatty marrow and Haversian canals (Onji et al., 1967). 
Although it may be merely another expression of degenerative change, ectopic bone formation differs from the usual secondary spondylotic calcification because: (1) it exceeds the anatomical limits of the posterior longitudinal ligament; (2) it is continuous with the vertebral bodies in contradistinction to the posterior longitudinal ligament which attaches only to the annulus; $(3)$ its presence is independent of the degree of spondylosis; and, (4) its predilection in the cervical spine is at a higher level than the usual spondylotic changes.

The presence or severity of neurological symptoms, particularly those of cord compression, depends on the relative width of the upper cervical spinal canal and the bulk of the cord. If the sagittal diameter of the canal is great and the cord relatively small, cord compression might not occur (case 2). On the other hand, formation of a wide layer of ectopic bone in a cervical canal, where the reserve space between the cord and its bony confines is small, will inevitably lead to severe compression (case 1). Associated bony spur formation encroaching on the intervertebral foramina could be responsible for the radicular symptoms, but a posterior displacement of the cord with stretching of the nerve roots might be a sufficient explanation. In our most pronounced case (case 1), the sagittal diameter of the spinal canal at the affected level was reduced to $11 \mathrm{~mm}$, as compared with the normal of $17.9 \mathrm{~mm}(16.0$ to 20.0$)$ (Payne, 1959). Whether this bone formation is progressive is not known, but eventually the formation of the voluminous slab of bone far exceeds the anatomical limits of the posterior longitudinal ligament itself. This seems to distinguish it from a mere calcium deposit within a normal-sized posterior longitudinal ligament which is seen as a manifestation of severe spondylosis of the cervical spine.

The aetiology of this ectopic bone formation is unknown. The factors that induce calls to form bone in unusual sites are poorly understood. Calcified matter of some sort seems to be essential (Ham and Harris, 1956). Ectopic ossification may occur practically anywhere in the body without any known metabolic disorder, although it is stated that ectopic bone cannot form in normal connective tissue. Interestingly, lime salts that deposit in new bone originate from the adjacent bone which thus becomes rarefied (Oppenheimer, 1942). In neither of our ectopic bone cases was there any rarefaction of the vertebral bodies, but in most of the second group the vertebral bodies were 'washed out'. Immobilization favours ossification, and this may explain why the bar occurs in the high cervical area where mobility is less. In case 2 , the anterior spurring, just below the inferior end of the bony bar, contributed undoubtedly to the immobility of the cervical spine at this level.

An infectious aetiology has been suggested (Cloward, 1967), implying that pharyngeal infection proceeds by the paravertebral venous plexus to the anterior spinal canal and there forms dystrophic calcification and eventually ossification. Neither our cases nor those reported elsewhere have shown evidence of infection. Interestingly, ossification of the lateral paravertebral ligaments with no degenerative changes in the spine occurs in psoriatic arthritis (Bywaters and Dixon, 1965) in which an infectious agent (PPLO) has been implicated (Wright and Reed, 1964). Ossification within the spinal canal has not been reported in this disease (Kaplan, Plotz, Nathanson, and Frank, 1963), but the subject is mentioned in passing in the connection of an infectious agent causing paravertebral ligamentous ossification.

Trauma has also been suggested as a cause of the ectopic ossification: small splinter fractures calcify and join posteriorly. However, this characteristically results in the confluence of posterior osteophytes and is not similar morphologically to the bony intraspinal bar. In Schneider's report of chronic cervical spinal trauma, there was bridging and calcification in the region of the anterior longitudinal ligament but no ossification within the spinal canal (Schneider, Papo, and Alvarez,1962).

About half of the cases reported by Onji et al. (1967) required surgical therapy. In their series, decompressive laminectomy had the best results. Our experience in case 1 attests to the fact that posterior decompression can be followed by a definite improvement. The experience of Onji et al. (1967) with anterior interbody fusion was disastrous. This is not surprising because adequate decompression cannot be carried out by this method. On the other hand, a piecemeal removal of the ossified structure through several intervertebral burr holes separated by parts of the vertebral bodies could hardly be carried out without injuring the already severely compromised cord or interfering with its blood supply through the anterior spinal artery.

\section{SUMMARY}

Seven cases of calcification and ossification within the spinal canal have been described. A distinction was attempted between ectopic ossification producing spinal cord compression as a clinical entity and degenerative calcification in the posterior longitudinal ligament as a secondary and usually asymptomatic manifestation of cervical spondylosis. Although they both may represent a different expression 
of the same underlying disease process, the former should be included in the differential diagnosis of cervical myelopathy because of its unique clinical behaviour. Decompressive laminectomy seems to be the treatment of choice.

\section{REFERENCES}

Bywaters, E. G. L., and Dixon, A. St. J. (1965). Paravertebral ossification in psoriatic arthritis. Ann. rheum. Dis., 24, 313-331.

Cloward, R. (1967). Personal communication.

Ham, A. W., and Harris, W. R. (1956). Repair and transplantation of bone. In: Biochemistry and Physiology of Bone. P. 504. Edited by Geoffrey H. Bourne. Academic Press: New York.

Kaplan, D. A., Plotz, C. M., Nathanson, L., and Frank, L. (1963). Cervical spine in psoriasis and psoriatic arthritis. Arthr. and Rheum., 6,281.
Minagi, H., and Gronner, A. T. (1969). Calcification of the posterior longitudinal ligament: A cause of cervical myelopathy. Amer. J. Roentgenol., 105, 365-369.

Onji, Y., Akiyama, H., Shimomura, Y., Ono, K., Hukuda, S., and Mizuno, S. (1967). Posterior paravertebral ossification causing cervical myelopathy. A report of eighteen cases. J. Bone Jt. Surg., 49A, 1314-1328.

Oppenheimer, A. (1942). Calcification and ossification of vertebral ligaments (spondylitis ossificans ligamentosa): Roentgen study of pathogenesis and clinical significance. Radiology, 38, 160-173.

Payne, E. E. (1959). The cervical spine and spondylosis. Neurochirurgia (Stuttg.), 1, 178-196.

Schneider, R. C., Papo, M., and Alvarez, C. S. (1962). The effects of chronic recurrent spinal trauma in high-diving. A study of Acapulco's divers. J. Bone Jt. Surg., 44A, 648-656.

Yokoi, K. (1963). Ectopic calcification in the epidural space. Orthop. Surg. (Tokyo), 14, 1262.

Wright, V., and Reed, W. B. (1964). The link between Reiter's syndrome and psoriatic arthritis. Ann. rheum. Dis., 23, 12-21. 